\title{
Segregation of HLA-DR2 among affected and non-affected offspring of 66 families with Type 1 (insulin-dependent) diabetes
}

\author{
I. Deschamps ${ }^{1}$, I. Goderel ${ }^{1}$, H. Lestradet ${ }^{1}$, M.Schmid ${ }^{2}$, M. Busson ${ }^{2}$, D. Cohen ${ }^{2}$ and J. Hors ${ }^{2}$ \\ ${ }^{1}$ Hôpital Herold and ${ }^{2}$ Hôpital Saint Louis, Paris, France
}

\begin{abstract}
Summary. Segregation of HLA-DR2 among affected and unaffected offspring was studied in 66 HLA-genotyped families with Type 1 diabetes in whom at least one parent carried DR2. The frequency of DR2-positive parents (21\%) was not different from that of control families (29\%). Among the diabetic probands, the gene frequency of DR2 was significantly decreased compared with control subjects $(0.05$ versus $0.17, p<$ $0.001)$ as were DR5 $(0.07$ versus $0.17, p<0.01)$ and DR7 $(0.06$ versus $0.13, p<0.003)$. Twenty probands carried DR2, in 11 of whom $(55 \%)$ it was found in combination with either DR3 or DR4. The nine cases who carried another DR allele included one who was DR2 homozygous. Transmission of DR2 was reduced in affected offspring, and random in unaffected siblings, compared with the expected ratio. However, when the
\end{abstract}

DR2 transmission was analysed separately for parents bearing DR2 with DR3, DR4 or another DR allele, it appeared that DR2 transmission to affected offspring was random when the parents carried neither DR3 or DR4, the transmission deficit being due to over-transmission of DR3 and DR4. The haplotype analysis showed that the haplotype A3, Cw7, B7, BfS, DR2, found in $19 \%$ of "non-diabetic" DR2 haplotypes was practically absent among "diabetic" DR2-haplotypes (4\%). In conclusion, population and segregation analysis could not demonstrate a specific protective effect of DR2.

Key words: Type 1 diabetes, haplotypes, heterogeneity, HLADR2 segregation.
A negative association between HLA-DR2 and Type 1 diabetes has been recognized in practically all populations and ethnic groups studied [1-10]. However, its significance is still unclear. It could be explained by a resistance gene in linkage disequilibrium with DR2 or in negative linkage disequilibrium with the susceptibility gene, or be merely secondary to the increased frequency of DR3 and DR4. Another hypothesis would be the heterogeneity of DR2, a subtype of which could confer resistance to Type 1 diabetes.

To date this problem has been poorly investigated $[11,12]$, possibly because of insufficient material, since most Type 1 diabetic series reported include very few or no DR2-positive patients. In this study we report a series of 66 families with Type 1 diabetes with at least one DR2-positive parent, in whom we have analysed the segregation of DR2 among diabetic and non-diabetic offspring.

\section{Subjects and methods}

In 171 out of 200 HLA-typed families ascertained through probands with Type 1 diabetes (onset before the age of 30 years), unequivocal
HLA-DR genotypes could be established in both parents and their offspring having obtained their informed consent. Sixty-six of these families were selected for the present study because at least one parent carried DR2. Their offspring included 66 probands with Type 1 diabetes ( 29 males and 37 females, mean age at onset 9.5 years), eight diabetic siblings and 91 unaffected siblings. Fifty-two normal families (104 unrelated parents) served as controls.

All subjects were typed for HLA-A, -B, -C, -DR and Bf following techniques described previously [3]. Gene and haplotype frequencies were obtained by direct counting. Linkage disequilibrium of the DR2 haplotype ( $\triangle$ and Ds) was calculated according to Dausset et al. [13].

\section{Results}

\section{Frequency of $D R 2$ in parents and probands}

Among the 342 parents of 171 families with Type 1 diabetes, 73 parents from 66 families carried DR2 (21\%). This frequency was not significantly different from that found in the 52 control families (29\%). Four DR2-positive and two DR2-negative parents in these families had Type 1 diabetes.

In seven families both parents were DR2-positive, one of whom was homozygous; in one family, one of 


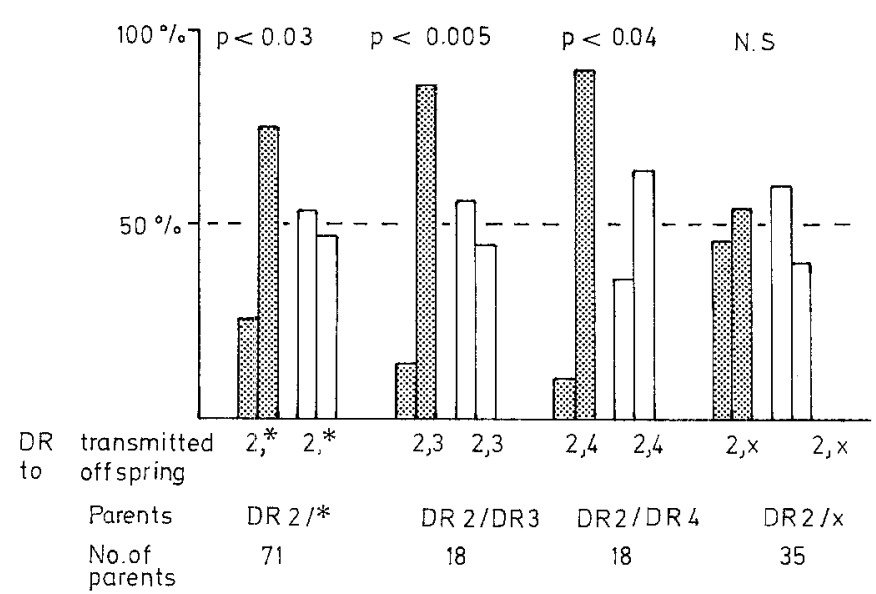

Fig. 1. The respective transmission of DR2 and the second DR-allele borne by the DR2-positive parents to affected (웅) and non-affected ( $\square$ ) offspring is shown for all DR2-positive parents, whatever the second DR-allele (DR2/*) and separately for parents bearing DR2/DR3, DR2/DR4, or DR2 combinations with alleles other than DR3 or DR4 $(D R 2 / x)$, $p$ values indicate statistical differences between affected and unaffected offspring

the parents was DR2 homozygous and in 58 families, one parent was DR2 heterozygous.

Twenty probands carried DR2 (seven males, $13 \mathrm{fe}$ males, mean age at onset of diabetes 6.9 years). The gene frequency $(0.05)$ was significantly lower than in the controls $(0.17, p<0.001 ; \mathrm{RR}: 0.27)$. DR5 and DR7 were also observed at lower frequencies $(0.07$ and 0.17 for DR5 in patients and control subjects respectively; $p<$ 0.01 ; RR: $0.30 ; 0.06$ and 0.13 for DR7, $p<0.003$; RR: $0.37)$.

DR2 was observed in the following allelic combinations: DR2/DR1 $(n=5)$, DR2/DR2 $(n=1)$; DR2/DR3 $(n=5)$; DR2/DR4 $(n=6)$; DR2/DR6 $(n=1)$; DR2/ DR7 $(n=2)$. No serological heterogeneous pattern of reactivity was observed in patients using local DR2 alloantibodies. Analysis with sera of the 9th Workshop is in progress.

\section{Segregation of $D R 2$}

Among the offspring of six families in whom both parents were DR2 heterozygous, the frequency of DR2 was decreased in probands ( 1 out of $6 ; 17 \%$ ), and random in unaffected siblings (5 out of $7 ; 71 \%$ ) compared with the expected frequency of $75 \%$ for Mendelian transmission.

In 58 families with one DR2 heterozygous parent, DR2 was also less frequently transmitted to probands (17 out of $58 ; 29 \%)$ and diabetic siblings ( 2 out of 8 ; $25 \%$ ), but randomly transmitted to unaffected siblings ( 40 out of $73 ; 54 \%$ ) in comparison with the expected ratio of $50 \%(p<0.03)$.

To exclude the bias introduced by selection of Type 1 diabetic patients from DR3 and DR4-positive individuals, we analysed separately the transmission of DR2 for parents bearing DR2/DR3, DR2/DR4 and $\mathrm{DR} 2 / \mathrm{DRx}$, where $\mathrm{x}$ includes all DR alleles other than
DR3 and DR4 (Fig. 1). DR2/DR3 and DR2/DR4-positive parents more often transmitted DR3 and DR4 to affected offspring than DR2. On the other hand, parents who carried a second DR allele other than DR3 or DR4 transmitted DR2 randomly. Transmission of DR2 to unaffected siblings was random in all cases.

\section{DR2-haplotypes}

The haplotype segment B7, BfS, DR2 which is found in linkage disequilibrium in Caucasoid populations $(\Delta$ 0.03 , Ds 0.429 in our control subjects) was also in linkage disequilibrium among the Type 1 diabetic patients $(\Delta 0.018$, Ds 0.417$)$. However, the weaker linkage disequilibrium of the whole haplotype $\mathrm{A} 3, \mathrm{Cw} 7, \mathrm{~B} 7, \mathrm{BfS}$, DR2 in control subjects (Ds 0.24) was not observed in Type 1 diabetic patients (Ds 0.06).

We have compared the prevalence of this haplotype and its segment among DR2-positive 'diabetic' haplotypes (i. e. present in a patient) and 'non-diabetic' haplotypes (i.e. present only in non-affected individuals) of our families with Type 1 diabetes. The segment B7, BfS, DR2 was found in six out of 23 'diabetic' $(26 \%)$ and in 17 out of $54(31 \%)$ 'non-diabetic' haplotypes. However, the whole haplotype $\mathrm{A} 3, \mathrm{Cw} 7, \mathrm{~B} 7, \mathrm{BfS}, \mathrm{DR} 2$ present in ten out of $54(19 \%)$ of 'non-diabetic' DR2-positive haplotypes, was found in only one of the $23(4 \%)$ DR2-positive 'diabetic' haplotypes.

\section{Discussion}

In both population and family studies, the excess of DR3 and DR4 alleles among Type 1 diabetic patients is the main problem in answering the question of whether the deficit of DR2 is a specific one, i. e. due to a protective effect of this allele, or the non-specific counterpart of the increase of DR3 and DR4. In favour of this latter hypothesis is the observation that two other alleles (DR5 and DR7) were also found at lower frequencies, confirming the data of other investigators $[4,5,7,14]$, although the decrease of DR2 was the most significant allelic decrease in the present and in all other series except one [14].

On the other hand, the segregation analysis failed to demonstrate a specific distortion of DR2 transmission. DR2 was transmitted randomly not only to unaffected children, but also to those affected children whose parents carried DR2 in combination with alleles other than DR3 and DR4. Indeed, the reduced transmission of DR2 to affected offspring appeared, on the whole, to be due to the bias of over-transmission of DR3 and DR4 by parents bearing one of these alleles.

However, if neither population nor family analyses were able to demonstrate a specific protective effect of DR2, several lines of evidence did suggest that DR2 in Type 1 diabetes could be heterogeneous: (1) When DR2 was analysed in its haplotypic combinations, the whole 
haplotype $\mathrm{A} 3, \mathrm{Cw} 7, \mathrm{~B} 7, \mathrm{BfS}, \mathrm{DR} 2$ found in $19 \%$ of 'non-diabetic' DR2-positive haplotypes, was almost absent from patient haplotypes (one out of $23,4 \%$ ) (2). Serologic heterogeneity of DR2 has been suggested by the description of two splits showing a 'long' and 'short' pattern of reactivity [15]. We were not able to detect heterogeneity among our patients using local DR2 sera; this will need to be confirmed by testing patients and controls with sera from the 9th Workshop. (3) The Dw2 and Dw12 specificities carried by most DR2-positive normal subjects were absent in the Type 1 diabetic patients of two studies $[1,11]$. However, this was not confirmed in another series [4]. Therefore, the possibility of a 'protective' DR2-Dw2 haplotype [12] remains open to question. (4) DNA restriction fragment analysis with a $\beta$-DC probe in DR2-positive individuals [16] has demonstrated heteroteneity of this genetic region, as a fragment present in control subjects and patients with multiple sclerosis was lacking in all Type 1 diabetic patients studied. Confirmation of this finding would allow a distinction to be made between 'protective' and 'nonprotective' DR2 haplotypes. More studies are needed to determine whether this fragment is correlated with one of the above-mentioned hypothetical variants. The mechanism by which the supposed 'non-protective' variant of DR2 confers susceptibility to Type 1 diabetes is still a matter of speculation.

In the case of the combination of DR2 with DR3 and DR4, a one-dose mechanism of susceptibility can be assumed. However, nine out of 20 patients $(45 \%)$ in our series carried neither DR3 nor DR4, and among these one DR2-homozygous patient is worth mentioning. The question of whether they represent a heterogeneous group from a pathogenetic point of view remains open. The hypothesis proposed by Bach et al. [12], assuming the involvement of a resistance factor very frequently associated with DR2 but rarely with DR3 and DR4, could account for the present results.

\section{References}

1. Barbosa J, Bach FH, Rich SS (1982) Genetic heterogeneity of diabetes and HLA. Clin Genet 21: 25-32

2. Cambon de Mouzon A, Ohayon E, Hauptmann G, Sevin A, Abbal M, Sommer E, Vergues H, Ducos J (1982) HLA-A, B, C, DR antigenes, Bf, C4 and glyoxalase I (GLO) polymorphisms in French Basques with insulin-dependent diabetes mellitus. Tissue Antigens 19: 366-379
3. Deschamps I, Lestradet H, Bonaiti C, Schmid M, Busson M, Benajam A, Marcelli-Barge A, Hors J (1980) HLA-genotype studies in juvenile insulin-dependent diabetes. Diabetologia 19: 189-193

4. Platz P, Jakobsen BK, Morling N, Ryder LP, Svejgaard A, Thomsen M, Christy M, Kromann H, Benn J, Nerup J, Green A, Hauge $M$ (1981) HLA-D and DR antigens in genetic analysis of insulindependent diabetes mellitus. Diabetologia 21: 108-115

5. Wolf E, Spencer KM, Cudworth AG (1983) The genetic susceptibility to Type 1 (insulin-dependent) diabetes: analysis of the HLADR association. Diabetologia 24: 224-230

6. Scholz S, Albert E (1983) HLA and disease: involvement of more than one HLA-linked determinant of disease susceptibility. Immunol Rev 70: 77-88

7. Murphy CC, Acton RT, Barger BO, Go RCP, Kirk KA, Reitnauer PJ, Roseman JM (1983) Population genetic analysis of insulin-dependent diabetes mellitus using HLA allele frequencies. Clin Genet 23: 405-414

8. Rodey GE, White N, Frazer TE, Duquesnoy RJ, Santiago JV (1979) HLA-DR specificities among black Americans with juvenile-onset diabetes. New Engl J Med 301: 810-812

9. Lee TD, Zhao T, Chi Z, Wong H, Shen M, Rodey G (1983) HLA$A, B$ and DR phenotypes in mainland Chinese patients with diabetes mellitus. Tissue Antigens 22:92-95

10. Sakurami T, Uemo Y, Nagaoka S, Iwaki Y, Park MS, Terasaki PI (1982) HLA-DR specifications in Japanese with juvenile-onset insulin-dependent diabetes mellitus. Diabetes 31: 105-106

11. Ilonen J, Herva E, Tiilikainen A, Akerblom HK, Koivukangast T, Kouvalainen K (1978) HLA-Dw2 as a marker of resistance against juvenile diabetes mellitus. Tissue Antigens 11: 144-146

12. Bach FH, Segall M, Rich S, Barbosa J (1982) HLA and susceptibility to Type 1 diabetes. Hypothesis. Tissue Antigens 20:28-32

13. Dausset J, Legrand L, Lepage V, Contu L, Marcelli-Barge A, Wildloecher I, Benajam A, Meo T, Degos L (1978) A haplotype study of HLA-complex with special reference to the HLA-DR series and to $\mathrm{Bf}, \mathrm{C} 2$ and GLO polymorphisms. Tissue Antigens 12: $297-307$

14. Serrano-Ríos M, Regueiro JR, Severino R, Lopez-Larrera C, Arnaiz-Villena A (1983) HLA-antigens in insulin-dependent and non-insulin-dependent Spanish diabetic patients. Diabete Metab 9: $116-120$

15. Richiardi P, Belvedere M, Borelli I, De Marchi M, Curtoni E (1978) Split of HLA-DRw2 into subtypic specificities correlated to two HLA-D products. Immunogenetics 7:57-61

16. Cohen D, Cohen O, Marcadet A, Massart C, Lathrop M, Deschamps I, Hors J, Schuller E, Dausset J (1984) HLA class II B DC DNA restriction fragments differentiate among HLA-DR2 individuals in insulin-dependent diabetes and multiple sclerosis. Proc Natl Acad Sci, USA (in press)

\author{
Dr. I. Deschamps \\ INSERM U 83 \\ Hôpital Herold \\ 7 place Rhin et Danube \\ F-75935 Paris Cedex 19 \\ France
}

